\title{
Inteligencia emocional y resiliencia en estudiantes universitarios, durante la pandemia del COVID-19
}

Emotional intelligence and resilience of university students, during the

$$
\text { COVID-19 pandemic }
$$

1 Alejandra Lizbeth Buenaño Salinas

https://orcid.org/0000-0001-7114-1829

Universidad Técnica de Ambato, Facultad de Ciencias de la Educación, Carrera de Psicopedagogía. Ambato, Ecuador abuenano7655@uta.edu.ec

2 Wilma Lorena Gavilanes López https://orcid.org/ 0000-0002-2563-6633

Universidad Técnica de Ambato, Facultad de Ciencias de la Educación, Carrera de Psicopedagogía. Ambato, Ecuador wilmalgavilanes@uta.edu.ec

3 Lenin Fabián Saltos Salazar

Universidad Técnica de Ambato, Facultad de Ciencias de la Educación, Carrera de Psicopedagogía. Ambato, Ecuador

lf.saltos@uta.edu.ec

4 Yanet Díaz Martín

Universidad Técnica de Ambato, Facultad de Ciencias de la Educación, Carrera de Psicopedagogía. Ambato, Ecuador ya.diaz@uta.edu.ec

Artículo de Investigación Científica y Tecnológica Enviado: 24/12/2021

Revisado: $29 / 12 / 2021$

Aceptado: $12 / 01 / 2022$

Publicado:08/03/2023

DOI: https://doi.org/10.33262/concienciadigital.v6i1.4.2054

Cítese:

Buenaño Salinas, A. L., Gavilanes López, W. L., Saltos Salazar, L. F., \& Díaz Martín, Y. (2023). Inteligencia emocional y resiliencia en estudiantes universitarios, durante la pandemia del COVID-19. ConcienciaDigital, 6(1.4), 1079-1097. https://doi.org/10.33262/concienciadigital.v6i1.4.2054

CONCIENCIA DIGITAL, es una Revista Multidisciplinar, Trimestral, que se publicará en soporte electrónico tiene como misión contribuir a la formación de profesionales competentes con visión humanística y crítica que sean capaces de exponer sus resultados investigativos y científicos en la misma medida que se promueva mediante su intervención cambios positivos en la sociedad. https://concienciadigital.org

La revista es editada por la Editorial Ciencia Digital (Editorial de prestigio registrada en la Cámara Ecuatoriana de Libro con No de Afiliación 663) www.celibro.org.ec 
Palabras claves: emociones, inteligencia emocional, resiliencia, estudiantes universitarios.

Keywords: emotions, emotional intelligence, resilience, university students.
Resumen

Debido a que las emociones ejercen gran influencia en la salud física y mental, varios investigadores han enfocado sus trabajos en cómo regular los estados emocionales para facilitar la resolución de conflictos y la adaptación al entorno. Objetivo: El presente estudio establece la relación entre inteligencia emocional y resiliencia en los estudiantes de nivelación de la Facultad de Ciencias Humanas y de la Educación de la Universidad Técnica de Ambato. Metodología: La investigación tiene un enfoque mixto, nivel descriptivo correlacional y modalidad documental, bibliográfica y de campo modalidad virtual. Aportes: Realizado el análisis y la interpretación, los resultados muestran que las dimensiones de inteligencia emocional claridad, atención y reparación se correlacionan con las dimensiones de resiliencia: satisfacción personal, ecuanimidad, confianza en sí mismo, sentirse bien sólo, perseverancia, pues obtienen una significancia bilateral menor a 0,05. Conclusión: Predominó el nivel adecuado de inteligencia emocional, seguido del bajo y del excelente. De igual manera predominó el nivel alto de resiliencia. No se encontraron diferencias significativas en cuanto al género y edad y se comprueba que la inteligencia emocional influye en la resiliencia de los estudiantes de la Universidad Técnica de Ambato.

\section{Abstract}

Because emotions exert great influence on our lives, several researchers have focused their work on how we regulate our emotional states to facilitate conflict resolution and adaptation to the environment. Objective: The present study to establish the relationship between emotional intelligence and resilience in the leveling students of the Faculty of Human Sciences and Education of the Technical University of Ambato. Methodology: The research has a mixed approach, correlational descriptive level and documentary, bibliographic and field modality virtual modality. Contributions: After analysis and interpretation, the results show that both the dimensions of emotional intelligence (clarity, attention and repair) correlate with the dimensions of resilience (personal satisfaction, equanimity, self-confidence, feeling good alone, perseverance), as they obtain a bilateral significance of less than 0.05. Conclusion: It is concluded that predominated the appropriate level of emotional intelligence. Similarly predominated high level 
of resilience. No differences were found in terms of gender and age. In conclusion emotional intelligence influences the resilience of students from the Technical University of Ambato.

\section{Introducción}

A menudo, los seres humanos no consideran el rol relevante que desempeñan las emociones en la vida diaria. Entender cómo funcionan las emociones, entender los sentimientos y cómo se sienten los demás es fundamental para la interacción con el mundo Dotar de inteligencia a las emociones resulta un verdadero reto. Ser inteligentes emocionalmente implica aplicar habilidades personales como la conciencia de uno mismo, autogestión, empatía y manejo de relaciones sociales (Goleman, 2018).

El adecuado manejo de las emociones involucra la constante interacción de procesos psíquicos. Una persona emocionalmente inteligente tiene la capacidad de regular sus estados emocionales, de modo que se le facilita la resolución de conflictos y la adaptación a su entorno. Conocer el funcionamiento de las emociones permitirá tomar el control de los impulsos, acciones que favorezcan el bienestar social, familiar y académico favor de la resiliencia.

Ser resiliente no es otra cosa que sobreponerse con éxito ante situaciones difíciles. La resiliencia contribuye al desarrollo de destrezas necesarias para enfrentar cualquier adversidad que se presenta en la vida. Aquel ser resiliente afronta de modo distinto los problemas, con un enfoque de oportunidad y aprendizaje.

Es preciso mencionar las palabras de Frankl (1979) en su libro "El hombre en busca de sentido", pues sostiene que en esta vida se puede perder todo, excepto la actitud con la que se enfrentan determinadas situaciones.

Las personas resilientes no permiten que sus emociones tomen el control, no son impulsivas, reconocen la importancia del autoconocimiento y en lugar de ver una dificultad, ven oportunidades de crecimiento y desarrollo personal.

Es importante indicar que al entender la manera en cómo se gestiona y se regula los estados emocionales, se maneja, afronta y supera situaciones complejas, producto de las exigencias del entorno. La pandemia del Covid-19 es un claro ejemplo de cómo se desarrolló esta capacidad. Como respuesta a la situación de la pandemia global (distanciamiento social, educación virtual, pérdidas familiares, despidos laborales, bajos ingresos económicos entre otros) corresponde generar respuestas adaptativas en torno a la inteligencia emocional y a la resiliencia en estudiantes universitarios.

Los objetivos del presente estudio se centran en describir la Inteligencia emocional según sus niveles y dimensiones, a continuación, identificar los niveles de resiliencia y sus 
enfoques. Finalmente determinar si las variables de género y edad influyen en los niveles de inteligencia emocional y resiliencia.

La educación es un proceso que orienta y da respuesta a la diversidad de los individuos. La declaración mundial de educación superior, precedida por la Organización para las Naciones Unidas para la Educación, Ciencia y Cultura (UNESCO, 1998), establece que los institutos de educación superior deberían promover la capacidad de los estudiantes para transformarse y provocar el cambio en medio de la incertidumbre, atender las necesidades sociales con fomentar la solidaridad. Para ello se vuelve necesario educar a los estudiantes universitarios en habilidades emocionales como la consciencia, regulación, autonomía emocional y empatía.

De igual forma, la convención mundial sobre el reconocimiento de las cualificaciones relativas a la educación superior y las convenciones regionales de educación superior precedida por la UNESCO (2020), establece que las instituciones de educación superior les corresponde un rol importante en la consecución de objetivos de desarrollo sostenible, haciendo énfasis en el objetivo 3 el cual comprende la salud y el bienestar de hombres y mujeres. En consecuencia, el fortalecimiento del desarrollo biopsicosocial de los universitarios resulta imprescindible para lograr una formación académica integral. Este acuerdo forja una nueva visión del contexto universitario, donde el desarrollo de competencias emocionales y resilientes constituye un punto de referencia dinámico que permita hacer frente a desafíos de la sociedad.

En el contexto ecuatoriano, se destaca el Plan Nacional de Desarrollo (PND) 2017-2021 Toda una Vida, el cual señala su primer objetivo la garantía de una vida digna con igualdad de oportunidades a través del acceso a una educación de calidad (SENPLADES, 2017). Así pues, la calidad educativa incluye la formación de profesionales con capacidad de afrontar a las necesidades del medio. Dicha formación se basa en aspectos de su bienestar personal y social.

Por su parte, los conceptos de inteligencia emocional y resiliencia surgieron dentro de los ámbitos de estudio de la Psicología Positiva, la cual se enfoca en las cualidades humanas y rasgos positivos que permiten lograr un estado de bienestar (García, 2017). Las emociones constituyen una parte inherente a los seres humanos, pues, cumplen un rol fundamental en el desarrollo de destrezas emocionales y sociales y que son decisivas para el aprendizaje y la adaptación a ciertas circunstancias que se presentan en la vida como hechos adversos (Manuel \& Mikel, 2017).

En principio, Salovey y Mayer propusieron el término Inteligencia Emocional (IE), basados en las inteligencias intrapersonal e interpersonal de Gardner. A partir de ello, sostienen que IE es aquella habilidad implicada en el manejo y empleo oportuno de las emociones y sentimientos (Postigo, 2015). Sin embargo, Goleman (2018) es quien 
difunde este concepto a través de su best-seller mundial "Inteligencia Emocional" donde indaga aspectos sobre la utilidad de las emociones, su naturaleza y muestra una serie de habilidades para una adecuada adaptación al entorno.

Goleman (2018) define a la inteligencia emocional como aquella capacidad de automotivarse, ser perseverante ante posibles frustraciones, control de impulsos, regulación de los estados de ánimo, empatía y confianza con los demás (Nieto, 2012). Ser emocionalmente inteligentes implica tener automotivación en condiciones de adversidad. Asimismo, el autocontrol y regulación de estados emocionales, significa entender lo que siente otra persona, lo que es esencial para interactuar con el mundo, de allí la necesidad de la educación emocional.

La educación emocional ha adquirido cierta relevancia en los últimos años. Esto, debido a que existe una creciente necesidad de que los planes de estudio ayuden a fomentar el desarrollo de competencias emocionales. La educación emocional como un continuo proceso educativo que busca potenciar el desarrollo emocional como componente imprescindible del desarrollo cognitivo, constituyendo ambos los elementos esenciales del desarrollo de la personalidad (Isidro \& Muriel, 2020).

Evidentemente, las emociones desempeñan un rol fundamental en la vida diaria, por lo tanto, para lograr una educación en emociones, resulta indispensable promover habilidades y conocimientos acerca de las emociones con la finalidad de dotar de herramientas que permitan afrontar las exigencias de la vida cotidiana.

En los últimos años, el término "Resiliencia" ha asumido un rol influyente en investigaciones de todo ámbito. Dicho concepto aparece por primera vez en el área de la ingeniería asociada con los conceptos de resistencia y elasticidad al rompimiento de materiales expuestos a la tensión formada por una determinada fuerza. (DGPOLDES, 2018). Sin embargo, no es hasta principios de los años 70 cuando la psicología positiva empezó un estudio de niños, provenientes de familias marginadas inmersas en la indigencia y trastornos mentales. Frente a esto, las investigadoras consideraron que niños desfavorecidos tenían algún trastorno. Sin embargo, se comprobó que un cierto porcentaje de infantes había alcanzado un desarrollo integral, fueron adultos competentes y tenían una vida normal. Aquellos niños que eran vulnerables y lograron sobreponerse los llamaron resilientes y, como consecuencia, se adoptó el nuevo término (Flores, 2021).

El término resiliencia es empleado en psicología para referirse a aquellas personas que nacen o viven en situaciones adversas, y que a pesar de ello se desarrollan psicológicamente sanas y exitosas (Muñoz, 2021). Lo que caracteriza a una persona resiliente es: Empatía, afecto, habilidad para comunicarse, sentido del humor y capacidad de respuesta social; habilidad para resolver problemas, elaborar estrategias, solicitar 
ayuda, creatividad, sentido crítico, autoeficacia; autonomía, sentido de identidad, autoconocimiento (Melendrez \& Ponce, 2019, pp. 9-10).

Evidentemente, todas estas características son esenciales para el desarrollo de competencias personales y sociales necesarias para la vida. De modo que la resiliencia constituye en sí, un rasgo inherente del ser humano que le otorga respuestas adaptativas ante situaciones difíciles.

La resiliencia promueve la calidad de vida puesto que permite enfrentar el estrés y los contratiempos. La resiliencia reduce la intensidad del estrés y los índices emocionales negativos (ansiedad, depresión y cólera) y, al mismo tiempo, aumenta la curiosidad y la salud emocional (Cyrulnik \& Anaut, 2016). Desde esta perspectiva, se puede considerar esencial para el desarrollo de la inteligencia emocional. El manejo de las emociones y el control del estrés resultan habilidades esenciales de interacción con el entorno.

La llegada del COVID 19 exigió cambios en los hábitos cotidianos, con efectos contraproducentes en las emociones. Muchas personas se han enfrentado a la ansiedad miedo y tristeza, razón por la cual se propone el uso de la inteligencia emocional y resiliencia como herramientas de apoyo (Fonseca, 2020). Estas capacidades inciden en la conducta, tanto al afrontar cualquier percance, como en la autogestión de los estados emocionales. Es decir, tener en claro una crisis y direccionarla con un enfoque resiliente e inteligencia emocional, puede contribuir a salir adelante como sociedad.

Cejudo (2016) en su investigación sobre la influencia en la satisfacción con la vida en estudiantes universitarios, sostienen que aquellas personas que tienen la capacidad para regular y controlar sus propios estados emocionales de forma adecuada gestionan mejor sus problemas y se sienten más satisfechos personalmente. Así mismo Estrada (2020) en su trabajo relacionado con las variables de Inteligencia emocional y resiliencia en adolescentes, establece que los estudiantes que presenten altos niveles de Inteligencia emocional serán más resilientes y podrán desarrollar respuestas adaptativas a adversidades.

\section{Metodología}

Se desarrolló una investigación con un enfoque mixto resultante de lo cuantitativo y cualitativo, pues fue un proceso que recolectó, analizó y utilizó datos cuantitativos y cualitativos en un mismo estudio con el análisis de los tests aplicados.

El diseño fue no experimental y los niveles o tipo descriptivo, porque describió las dos variables del estudio y las características de personas con un elevado nivel de inteligencia emocional y resiliencia. También fue de tipo correlacional debido a se estableció la relación o asociación de las variables de estudio. 
Utilizó la modalidad documental, pues se recopiló datos en base a recursos bibliográficos. Además, se desarrolló en modalidad de campo virtual, debido a que recogió y registró ordenadamente los datos relativos al objeto de estudio en base a instrumentos de evaluación trasladados a Google Forms para facilitar la recolección de información.

La población y la muestra fue de 212 estudiantes de nivelación de la facultad de ciencias humanas y de la educación de la Universidad Técnica de Ambato. De ellos 148 son del sexo femenino y 68 del masculino con edades de los 17 a los 18 años y que de acuerdo con los criterios de selección de la muestra dieron su consentimiento informado, lo cual permitió contar con la participación libre y voluntaria en esta investigación.

Como instrumentos para la recolección de los datos se hizo la aplicación de tipo colectiva mediante la plataforma Zoom y los dos formularios en Google Forms de los siguientes tests psicométricos:

El primer test fue la Escala Rasgo de Metaconocimiento Emocional (Escala TMMS-24) con la finalidad de evaluar la inteligencia emocional intra percibida. Posee tres dimensiones o subescalas: atención emocional (percepción de los sentimientos de forma adecuada) claridad emocional (compresión de estados emocionales) y reparación emocional (regular los propios estados emocionales). La escala se compone de 24 ítems que deben ser puntuados con una escala tipo Likert de cinco puntos (desde 1= Nada de acuerdo, hasta 5=Totalmente de acuerdo). La prueba Alfa de Cronbach determina un nivel de confiabilidad del 0,924 (Lalaleo, 2020).

De igual manera se aplicó la escala de resiliencia de Wagnild y Young. Su aplicación fue de tipo colectiva. La finalidad de la prueba fue evaluar el nivel de adaptación psicosocial frente a eventos de la vida, a través de cinco dimensiones: Ecuanimidad, sentirse bien solo, confianza en sí mismo, perseverancia y satisfacción personal. Esta escala se compone de 25 ítems, los cuales puntúan en una escala de tipo Likert en donde 1 es "en desacuerdo" y 7 es "de acuerdo". La consistencia interna, derivada a partir de la prueba estadística alfa de Cronbach, determina un nivel de confiabilidad del 0,93 (Montero \& Lara, 2018)

Una vez recopilados los datos, el análisis se realizó mediante el programa Paquete Estadístico para ciencias sociales (SPSS del inglés Statistical Package for the Social Sciences), en el cual se utilizó la prueba no paramétrica de Rho de Spearman para comparar dos muestras independientes, también se utilizó la prueba t para muestras independientes para identificar la asociación entre edad, sexo y el nivel de inteligencia emocional y resiliencia. 


\section{Resultados}

La tabla 1 detalla la población conformada por 212 estudiantes, que cursan nivelación en la facultad de ciencias humanas y de la educación de la Universidad Técnica de Ambato, correspondiente al semestre abril a septiembre 2021.

Del total de la población, se observa un predominio del género femenino respecto al masculino con un $70 \%$ del total. Concerniente a la edad, el rango de mayor incidencia es de 17 a 22 años, con un $75 \%$ del total.

\section{Tabla 1}

Información sociodemográfica

\begin{tabular}{cccc}
\hline \multirow{3}{*}{ Género } & & Frecuencia & $\%$ \\
& Femenino & 148 & $70 \%$ \\
\multirow{3}{*}{ Edad } & Masculino & 64 & $30 \%$ \\
& De 17 a 22 & 159 & $75 \%$ \\
& De 23 a 28 & 53 & $25 \%$ \\
& Total & 212 & $100 \%$
\end{tabular}

Fuente: Datos obtenidos de la aplicación de test en Google Forms

A continuación, se presenta la tabla 2 sobre la descripción de los resultados obtenidos para cada variable estudiada.

\section{Tabla 2}

Distribución de frecuencias de niveles de inteligencia emocional

\begin{tabular}{ccccc}
\hline & Bajo & Adecuado & Excelente & Total \\
\hline Atención emocional & $34 \%$ & $53 \%$ & $13 \%$ & $100 \%$ \\
Claridad emocional & $37 \%$ & $53 \%$ & $10 \%$ & $100 \%$ \\
Reparación emocional & $26 \%$ & $56 \%$ & $18 \%$ & $100 \%$ \\
\hline
\end{tabular}

Fuente: Datos obtenidos de la aplicación de test en Google Forms

En la dimensión Atención emocional se encontró que presentan un nivel bajo ya que el $34 \%$ de estudiantes presta poca atención a los sentimientos, el 53\% posee una adecuada atención a sus sentimientos y el $13 \%$ prestan demasiada atención a sus sentimientos ubicados en un nivel excelente.

Los resultados obtenidos manifiestan que más del $50 \%$ de estudiantes poseen una adecuada atención a sus sentimientos, es decir que poseen capacidad para percibir y sentir sus emociones. Asimismo, consideran importante prestar atención a sus estados de ánimo para que sus sentimientos y emociones no interfieran en sus pensamientos y acciones 
cotidianas. Además, al dedicar tiempo a sus emociones, desarrollan la habilidad de reconocer sentimientos e interpretar su significado, con el fin de contribuir a su desarrollo personal.

En la dimensión de claridad emocional, se evidencia que el $37 \%$ de estudiantes deben mejorar su claridad emocional, el 53\% de estudiantes presentan adecuada claridad emocional y el $10 \%$ de estudiantes, posee excelente claridad emocional.

Estos resultados demuestran que la mayoría de los estudiantes poseen adecuada claridad emocional, es decir que no tienen dificultades para conocer y comprender sus propias emociones. Por tal motivo definen sus sentimientos, conocen cómo se sienten y en diferentes situaciones pueden expresar cuáles son sus emociones, se destaca la habilidad de comprender sus propios estados emocionales y los de los demás.

En la dimensión de reparación de las emociones, el 26\% de evaluados deben mejorar su reparación emocional, el 56\% presentan adecuada reparación emocional y el 18\% poseen excelente reparación emocional. De los resultados obtenidos, se observa que gran parte de los estudiantes poseen adecuada reparación emocional, por consiguiente, se destacan por tener la capacidad de regular sus propios estados emocionales a través del pensamiento positivo, visión optimista y un enfoque de calma ante los problemas. De igual manera, buscan control de las emociones positivas y negativas para fomentar el desarrollo de la autoestima y empatía.

Tabla 3

Distribución de frecuencias de niveles de resiliencia

\begin{tabular}{lcccc}
\hline & Bajo & Moderado & Alto & Total \\
\hline Satisfacción personal & $4 \%$ & $29 \%$ & $67 \%$ & $100 \%$ \\
Ecuanimidad & $5 \%$ & $43 \%$ & $52 \%$ & $100 \%$ \\
Sentirse bien sólo & $6 \%$ & $26 \%$ & $68 \%$ & $100 \%$ \\
Confianza en sí mismo & $7 \%$ & $27 \%$ & $66 \%$ & $100 \%$ \\
Perseverancia & $4 \%$ & $32 \%$ & $64 \%$ & $100 \%$ \\
\hline
\end{tabular}

Fuente: Datos obtenidos de la aplicación de test en Google Forms

En cuanto a la variable resiliencia en los resultados obtenidos se identifica que, en la dimensión de satisfacción personal, el $67 \%$ obtiene una puntuación alta, 29\% moderado y $4 \%$ bajo, lo que evidencia que gran parte de los estudiantes alcanzan un nivel alto en la satisfacción personal, de tal forma que entienden el significado de su vida, analizan, comprenden sus contribuciones y propósitos relacionados a su calidad de vida. Esto puede explicarse por la etapa evolutiva de ser adultos jóvenes los sujetos del estudio. 
Por otra parte, en la dimensión de ecuanimidad, el 52\% obtiene una puntuación alta, 43\% moderado y $5 \%$ bajo. Se evidencia que mayoría de la población alcanza un nivel alto, es decir que poseen una visión equilibrada de las experiencias de vida y no valoran negativamente un acontecimiento. Asimismo, contribuyen a su bienestar generando respuestas adaptativas ante la adversidad.

En cuanto a la dimensión de sentirse bien sólo, el 68\% una puntuación alta, 26\% moderado y $6 \%$ bajo Un amplio porcentaje del total de la población es consciente que, a pesar de las experiencias compartidas, como seres humanos deben enfrentarse solos a ciertas situaciones, con un enfoque de autonomía y autenticidad.

Mientras que para la dimensión de confianza en sí mismo, el 66\%, de la población objeto de estudio puntúa alto, $27 \%$ moderado y $7 \%$ bajo. Dicho de otra manera, gran parte de los estudiantes cree en sus capacidades de logro y desarrollan independencia, es decir, dependen de sí mismos para el alcance de metas y objetivos. De igual manera poseen la capacidad de identificar sus fortalezas y debilidades.

Finalmente, en la dimensión de perseverancia el $64 \%$ obtiene una puntuación alta, 32\% moderado y $4 \%$ bajo. En efecto, un porcentaje significativo considera que el ser perseverante le asegurará firmeza en sus decisiones y propósitos, a pesar de las adversidades. Además, obtienen un aprendizaje de las dificultades de la vida en tanto que desarrollan autodisciplina y motivación.

Se presenta una selección de los resultados obtenidos en este trabajo, en cada análisis se utilizó datos estadísticos que fueron medidos por el programa SPSS.

\section{Tabla 4}

Estadísticos descriptivos y prueba t para muestras independientes en función del género en la escala TMMS-24 y escala de Wagnild y Young

\begin{tabular}{lllllllll}
\hline Dimensión & Género & $\mathrm{N}$ & $\mathrm{M}$ & $\mathrm{DT}$ & $\mathrm{t}$ & $\mathrm{p}$ \\
\hline Atención emocional & Femenino & 148 & 27,03 & 6,20 &, 15 &, 882 \\
& Masculino & 64 & 26,89 & 6,99 &, 14 &, 888 \\
& Femenino & 148 & 25,88 & 6,70 &,- 12 &, 903 \\
Claridad emocional & Masculino & 64 & 26,00 & 6,54 &,- 12 &, 902 \\
& Femenino & 148 & 28,24 & 6,94 &, 47 &, 638 \\
Reparación emocional & Masculino & 64 & 27,77 & 6,05 &, 50 &, 620
\end{tabular}




\section{Tabla 4}

Estadísticos descriptivos y prueba t para muestras independientes en función del género en la escala TMMS-24 y escala de Wagnild y Young (continuación)

\begin{tabular}{llllllll}
\hline Dimensión & Género & N & M & DT & t & p \\
\hline Satisfacción personal & Femenino & 148 & 20,90 & 4,79 &, 50 &, 615 \\
& Masculino & 64 & 20,55 & 4,39 &, 52 &, 603 \\
Sentirse bien sólo & Femenino & 148 & 15,74 & 3,69 &,- 44 &, 657 \\
& Masculino & 64 & 15,98 & 3,49 &,- 45 &, 651 \\
Perseverancia & Femenino & 148 & 36,69 & 8,51 &,- 18 &, 860 \\
& Masculino & 64 & 36,91 & 7,57 &,- 18 &, 854 \\
Confianza en mí mismo & Femenino & 148 & 35,89 & 9,10 &, 36 &, 717 \\
& Masculino & 64 & 35,42 & 7,03 &, 40 &, 689 \\
& Femenino & 148 & 19,18 & 4,19 & $-1,31$ &, 193
\end{tabular}

Fuente: Datos obtenidos de la aplicación de test en Google Forms

En la tabla 4 se puede comprobar que las puntuaciones más altas en las distintas categorías están repartidas entre hombres y mujeres. La media de las puntuaciones de los hombres supera a la de las mujeres en claridad emocional, sentirse bien sólo, perseverancia y ecuanimidad, mientras que la media de las puntuaciones de las mujeres supera a la de los hombres en atención, reparación emocional, satisfacción personal y confianza en mí mismo.

Los resultados de la prueba t para muestras independientes en función del género no presentan diferencias estadísticamente significativas $(\mathrm{p}>0,05)$, pues en todas las dimensiones el valor $\mathrm{p}$ es mayor a 0,05 . 


\section{Tabla 5}

Estadísticos descriptivos y prueba t para muestras independientes en función de edad en la escala TMMS-24 y escala de Wagnild y Young

\begin{tabular}{|c|c|c|c|c|c|c|}
\hline Edad & & $\mathrm{N}$ & Media & DT & $\mathrm{T}$ & $\mathrm{p}$ \\
\hline \multirow{2}{*}{ Atención emocional } & De 17 a 22 & 159 & 26,89 & 6,45 &,- 381 & ,703 \\
\hline & De 23 a 28 & 53 & 27,28 & 6,22 &,- 382 & ,703 \\
\hline \multirow[t]{2}{*}{ Claridad emocional } & De 17 a 22 & 159 & 25,59 & 6,57 & $-1,233$ & ,219 \\
\hline & De 23 a 28 & 53 & 26,89 & 6,67 & $-1,212$ & ,229 \\
\hline \multirow[t]{2}{*}{ Reparación emocional } & De 17 a 22 & 159 & 27,91 & 6,88 &,- 712 & ,477 \\
\hline & De 23 a 28 & 53 & 28,16 & 5,39 &,- 760 & ,449 \\
\hline \multirow[t]{2}{*}{ Satisfacción personal } & De 17 a 22 & 159 & 20,87 & 4,8 &, 407 & ,684 \\
\hline & De 23 a 28 & 53 & 20,81 & 4,33 & ,432 & ,667 \\
\hline \multirow[t]{2}{*}{ Sentirse bien sólo } & De 17 a 22 & 159 & 15,82 & 3,69 & ,011 & ,991 \\
\hline & De 23 a 28 & 53 & 15,91 & 3,42 &, 011 & ,991 \\
\hline \multirow[t]{2}{*}{ Perseverancia } & De 17 a 22 & 159 & 36,5 & 8,53 &,- 771 & ,442 \\
\hline & De 23 a 28 & 53 & 37,56 & 7,52 &,- 835 & ,406 \\
\hline \multirow[t]{2}{*}{ Confianza en mí mismo } & De 17 a 22 & 159 & 35,53 & 7,45 &,- 642 &, 522 \\
\hline & De 23 a 28 & 53 & 36,81 & 12,04 &,- 528 & ,599 \\
\hline \multirow[t]{2}{*}{ Ecuanimidad } & De 17 a 22 & 159 & 19,23 & 4,25 & $-1,216$ & ,225 \\
\hline & De 23 a 28 & 53 & 20,05 & 3,66 & $-1,313$ & ,192 \\
\hline
\end{tabular}

Fuente: Datos obtenidos de la aplicación de test en Google Forms

La tabla 5 muestra los estadísticos descriptivos para los intervalos de edad en cada una de las dimensiones consideradas. Se puede observar que, en la mayoría de las dimensiones (atención, claridad, reparación emocional, sentirse bien sólo, perseverancia, confianza en sí mismo, ecuanimidad) los sujetos con una edad de 23 a 28 años obtienen la media más elevada. Solamente en el factor satisfacción personal la categoría de 17 a 22 años obtiene una media elevada. Los resultados de la prueba t para muestras independientes en función de la edad no presentan diferencias estadísticamente significativas ( $\mathrm{p}>0,05)$, pues en todas las dimensiones el valor $\mathrm{p}$ es mayor a 0,05 . 
Cabe destacar que en los resultados obtenidos de la prueba t para muestras independientes en función del género y edad no presentan diferencias estadísticamente significativas ( $\mathrm{p}$ $<0,05$ ), pues en todas las dimensiones el valor p es mayor a 0,05 (Ver tabla 10 y 11). Por tanto, el género y edad no influye significativamente en los niveles de inteligencia emocional y resiliencia.

Los resultados coinciden con el estudio de Castro et al. (2018), quienes no encontraron asociaciones estadísticas ( $\mathrm{p} \geq .05)$ al analizar en deportistas las variables de inteligencia emocional en función del sexo. Sin embargo, muestran relaciones estadísticamente significativas con el factor edad $(\mathrm{p} \leq .05)$, pues los deportistas mayores de 22 muestran mejor percepción y gestión emocional.

Finez et al. (2019) sostienen que existen diferencias significativas $(p \leq .05)$, en correspondencia a la edad y resiliencia entre personas de 16 a 18 años y de 26 a 45 años. Además, indican que el género no difiere significativamente $(\mathrm{p} \geq .05)$ en la resiliencia.

En contraste, se presenta el estudio de Sanmartín et al. (2018), quienes establecen las puntuaciones de la IE en función al género, e indican que el género masculino $(27,90)$ supera al género femenino $(24,23)$ en atención, claridad y reparación emocional. No obstante, Merchán et al. (2018), en su investigación sobre el valor predictivo de la inteligencia emocional rasgo en los estados afectivos: una comparación de género establece que los universitarios no manifiestan diferencias de género sobre la percepción de sus habilidades emocionales.

López et al. (2019) en su estudio sobre niveles de inteligencia emocional según género de estudiantes en la educación superior, manifiesta que, para el sexo masculino, las dimensiones Claridad y Reparación, presentan puntuaciones superiores a las alcanzadas por las mujeres, no obstante, en este género, en relación con la dimensión Atención, logra mayor puntuación que en los hombres.

En cuanto a resiliencia, un estudio realizado por Ponte (2017) sostiene que predomina en los estudiantes universitarios un nivel medio de resiliencia a nivel general y en sus dimensiones, con porcentajes que oscilan entre $37.6 \%$ y $49.6 \%$. De igual manera, Romero (2018) afirma que los adolescentes objeto de estudio, obtienen puntuaciones mayores en las dimensiones de Aceptación de uno mismo $(\mathrm{H}=34,23$ y $\mathrm{M}=34,56)$ y Ecuanimidad ( $\mathrm{H}=27,48$ y $\mathrm{M}=26,21)$. 


\section{Tabla 6}

Correlación Rho de Spearman entre dimensiones de variables

\begin{tabular}{|c|c|c|c|c|}
\hline & \multicolumn{4}{|c|}{ Inteligencia Emocional } \\
\hline & & Atención & Claridad & Reparación \\
\hline $\begin{array}{l}\text { Satisfacción } \\
\text { personal }\end{array}$ & $\begin{array}{l}\text { Coeficiente de } \\
\text { correlación }\end{array}$ &, $407 * *$ &, $406 * *$ &, $304 * *$ \\
\hline & Sig. (bilateral) &, 000 & ,000 &, 000 \\
\hline $\begin{array}{l}\text { Sentirse bien } \\
\text { sólo }\end{array}$ & $\begin{array}{l}\text { Coeficiente de } \\
\text { correlación }\end{array}$ &, $444 * *$ &, $397 * *$ &, $414^{* *}$ \\
\hline & Sig. (bilateral) & ,000 &, 000 &, 000 \\
\hline $\begin{array}{l}\text { Confianza en sí } \\
\text { mismo }\end{array}$ & $\begin{array}{l}\text { Coeficiente de } \\
\text { correlación }\end{array}$ &, $452 * *$ &, $403 * *$ &, $370 * *$ \\
\hline & Sig. (bilateral) &, 000 &, 000 &, 000 \\
\hline Perseverancia & $\begin{array}{l}\text { Coeficiente de } \\
\text { correlación }\end{array}$ &, $471 * *$ &, $430 * *$ &, $347 * *$ \\
\hline & Sig. (bilateral) &, 000 &, 000 &, 000 \\
\hline Ecuanimidad & $\begin{array}{l}\text { Coeficiente de } \\
\text { correlación }\end{array}$ &, $495^{* *}$ &, $423 * *$ &, $383 * *$ \\
\hline & Sig. (bilateral) &, 000 &, 000 &, 000 \\
\hline
\end{tabular}

**. La correlación es significativa al nivel 0,01 (bilateral).

Fuente. Datos obtenidos del programa SPSS

En cuanto a la asociación de la inteligencia emocional y la resiliencia se encontraron correlaciones significativas de atención emocional con satisfacción personal, sentirse bien sólo, confianza en sí mismo, perseverancia ecuanimidad (sig. ,000). En correspondencia con claridad emocional se encontraron asociaciones con satisfacción personal, confianza en mí mismo, ecuanimidad, sentirse bien solo y perseverancia (sig. ,000). La dimensión de reparación emocional posee asociaciones significativas con los factores satisfacción personal, sentirse bien sólo, confianza en sí mismo, perseverancia ecuanimidad (sig. ,000).

\section{Discusión}

A partir de los resultados obtenidos del análisis inferencial, se encontró asociación significativa (Sig 0,000), entre todas las dimensiones de Inteligencia Emocional y resiliencia, lo que afirma que la inteligencia emocional influye en la resiliencia de los 
estudiantes de nivelación de la Facultad de Ciencias Humanas y de la Educación de la Universidad Técnica de Ambato. Con los datos obtenidos del presente estudio, se concuerda con Estrada (2020), quien muestra que existe una relación directa y significativa entre la inteligencia emocional y la resiliencia ( $r s=0,676 ; p=0,000<0,05)$, es decir que cuánto más alto sea el nivel de inteligencia emocional, el nivel de resiliencia será mayor en los estudiantes.

Asimismo, la investigación de Cejudo (2016) relacionó de manera estadísticamente significativa y positiva la asociación de la satisfacción con la vida y la IE, de modo particular con claridad emocional $(r=0,26 ; p<0,01)$ y con reparación emocional $(r=$ $0,35 ; p<0,01)$. El trabajo investigativo manifiesta que, aquellas personas que tienen la capacidad para regular sus estados emocionales gestionan mejor sus problemas y sienten bienestar.

Del mismo modo, Meléndez et al. (2019) encontraron correlaciones significativas positivas entre inteligencia emocional y la resiliencia; se comprobó también que las dimensiones de claridad y regulación emocional predicen significativa y positivamente la resiliencia. Esto debido a que la claridad y regulación de las emociones fomentan la adaptación al envejecimiento y ayudan a comprender qué emociones se están percibiendo.

\section{Conclusiones}

- En cuanto a la inteligencia emocional se concluye que predominó el nivel adecuado en las dimensiones atención emocional (53\%), claridad emocional (53\%) y reparación emocional (56\%). En segundo lugar, se ubicó el nivel bajo y en tercer lugar se ubicaron los estudiantes con un nivel excelente de su inteligencia emocional.

- En cuanto a la resiliencia la mayoría de los estudiantes se ubicó en el nivel alto de oscilando el porcentaje de sus dimensiones del $57 \%$ al $68 \%$ por lo que predominó el nivel alto en las dimensiones: sentirse bien solo en el 68\%, satisfacción personal en el $67 \%$, confianza en sí mismo en el 66\%, perseverancia en el $64 \%$ y ecuanimidad en el $52 \%$ del total de estudiantes investigados.

- Los resultados obtenidos de la prueba t para muestras independientes en función del género y edad no presentan diferencias estadísticamente significativas en las variables estudiadas.

- Se estableció la relación entre inteligencia emocional y resiliencia mediante de los resultados obtenidos en la prueba estadística Rho de Spearman, en donde se obtuvo un nivel de significancia del menor al 0,05; que comprueba que Inteligencia emocional se relaciona con la resiliencia de los estudiantes de nivelación de la Facultad de Ciencias Humanas y de la Educación de la Universidad Técnica de Ambato. 
- De este modo pues, resulta imprescindible para la comunidad educativa ejecutar programas de asistencia integral encaminados fomentar la inteligencia emocional y resiliencia, pues el desarrollo de competencias emocionales permite hacer frente a situaciones de adversidad o conflicto, con el propósito de crear un ambiente de bienestar personal y social en los estudiantes.

\section{Referencias bibliográficas}

Castro, M, Zurita, F. \& Chacón, R. (2018). Inteligencia emocional en deportistas en función del sexo, la edad y la modalidad deportiva practicada. Science Technical Journal,4(1), 288-305

Cejudo. (2016). Inteligencia emocional y resiliencia: su influencia en la satisfacción con la vida en estudiantes universitarios. Anuario de Psicología, 46(2), 51-57.

Cyrulnik, B. \& Anaut, M. (2016). ¿Por qué la resiliencia? Lo que nos permite reanudar la vida. ¿Por qué la resiliencia? Barcelona, Editorial Gedisa.

DGPOLDES. (2018). Construcción de Resiliencia para el Bienestar. Directrices para la Cooperación Española Subdirección General de Planificación, Coherencia de Políticas y Evaluación. Dirección General de Políticas de Desarrollo Sostenible. Ministerio de Asuntos Exteriores y Cooperación

Estrada, E. (2020). Inteligencia emocional y resiliencia en adolescentes de una institución educativa pública de Puerto Maldonado. Ciencia y Desarrollo, 23(3), 28-35.

Finez, M., Moran, C. \& Urchaga, J. (2019). Resiliencia psicológica a través de la edad y el sexo. International Journal of Developmental and Educational Psychology,4(1), 85-95.

Flores, N. (2021). La importancia de la resiliencia en tiempos de Covid-19. Revista Conciencia Digital, 4(1.2), 269-285.

Fonseca, M. (2020). La resiliencia y la inteligencia emocional, claves en momentos de crisis. BBVA. https://www.bbva.com/es/co/la-resiliencia-y-la-inteligenciaemocional-claves-en-momentos-de-crisis/

García, E. (2017). Bienestar Emocional en Educación: Empecemos por los Maestros. (Tesis doctoral). Universidad de Murcia, Facultad de Psicología, Murcia, España.

Frankl, V. (1979). El hombre en busca de sentido. Editorial Herder S.A: Barcelona, España. 
Goleman, D. (2018). Aprendemos Juntos. Versión Completa. Los beneficios de la inteligencia emocional para nuestros hijos. [Video] https://www.youtube.com/watch?v=k6Op1gHtdoo\&t=34s

Isidro, A \& Muriel, M. (2020). Creciendo con las emociones: inteligencia emocional de la niñez a la adolescencia. International Journal of Developmental and Educational PsychologyINFAD Revista de Psicología, 2(1), 137-148.

Lalaleo, A. (2020). La Inteligencia Emocional y los Trastornos Alimenticios. [Tesis de pregrado]. Facultad de Ciencias Humanas y de la Educación de la Universidad Técnica de Ambato.

López. (2019). Niveles de inteligencia emocional según género de estudiantes en la educación superior. Revista Espacios, 40(31), 26.

Manuel, J. \& Mikel, J. (4 de mayo del 2017). Resiliencia e Inteligencia Emocional. Campuseducacion.com.

Meléndez, J., Delhmon, I \& Satorres, E. (2019). El poder de la inteligencia emocional sobre la resiliencia en adultos mayores. Ansiedad y Estrés, 25(1), 14-19.

Melendrez, Y. \& Ponce, C. (2019). Resiliencia en inmigrantes: características, factores e instrumentos. Una revisión teórica (Tesis de pregrado). Universidad Peruana Unión. Facultad de Ciencias de la Salud Escuela Profesional de Psicología, Chosica, Perú.

Merchán. (2018). Valor predictivo de la inteligencia emocional rasgo en los estados afectivos: una comparación de género. Revista de Psicología, 1(1), 137-146.

Montero, A. \& Lara, R. (2018) Dimensiones de la personalidad y niveles de resiliencia en pacientes de la Unidad Oncológica SOLCA DE Tungurahua. Pontificia Universidad Católica del Ecuador Sede Ambato.

Muñoz, J. (2021). Abordaje de la Resiliencia desde el proceso de atención de enfermería: A propósito de un caso. Rev. Enferm Salud Mental, 17, 22-26.

Nieto, A (2012). Inteligencia emocional. Universidad Virtual del Estado de Guanajuato, Guanajuato, México.

Organización para las Naciones Unidas para la Educación, Ciencia y Cultura [UNESCO]. (1998). Declaración Mundial de Educación Superior en el siglo XXI: Visión y Acción. Educación Superior y Sociedad, 9(2), 97-113

Organización para las Naciones Unidas para la Educación, Ciencia y Cultura [UNESCO]. (2020). Educación superior y Objetivos de Desarrollo Sostenible. 
Ponte. (2017). Adaptación a la vida universitaria y resiliencia en estudiantes de psicología de una Universidad Privada de Trujillo. [Tesis de grado]. Universidad Privada Antenor Orrego.

Postigo, O. (2015). Inteligencia emocional (tesis de grado). Universidad de Almería, Almería, España.

Romero. (2018). Diferencias por sexo en la intimidación escolar y la resiliencia en adolescentes. Psicología Escolar y Educacional, 22(3), 1-9.

Sanmartín, R., Gonzálvez, C. \&Vicent, M. (2018). Inteligencia emocional en alumnado de formación profesional. Diferencias en función del curso, del género y de la edad. Educar,54 (1), 229-245.

SENPLADES. (2017). Plan Nacional de Desarrollo. https://www.planificacion.gob.ec/wpcontent/uploads/downloads/2017/10/PNBV26-OCT-FINAL_0K.compressed1.pdf 
El artículo que se publica es de exclusiva responsabilidad de los autores y no necesariamente reflejan el pensamiento de la Revista Conciencia Digital.

\section{Ciencia \\ LDigital}

El artículo queda en propiedad de la revista y, por tanto, su publicación parcial y/o total en otro medio tiene que ser autorizado por el director de la Revista Conciencia Digital.
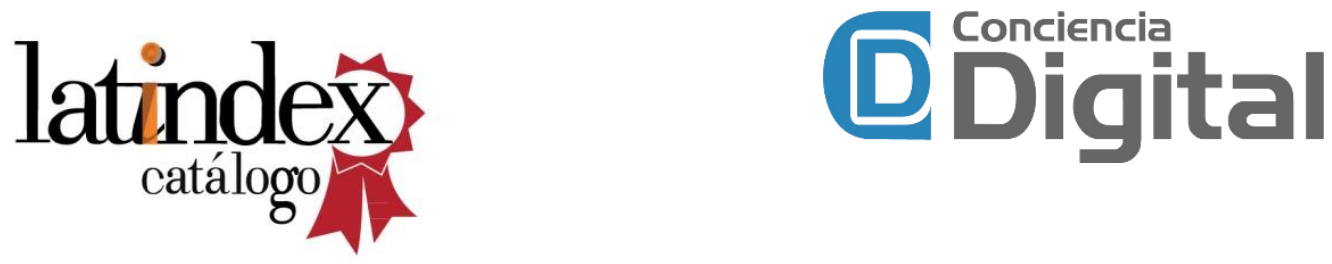

Indexaciones

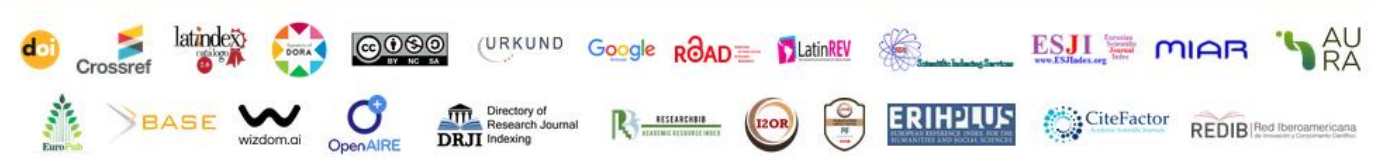

